\title{
WHY CONSUMERS SEEK ‘COOLNESS’? EVIDENCE FROM THE ARAB WORLD
}

Kaleel Rahman, RMIT University, Australia

Gaurangi Laud, RMIT University, Australia

\begin{abstract}
The charm of coolness is seemingly enhanced by the mysteriousness of what cool actually means. Figuring out how to be "cool" is arguably an important phenomenon amongst many modern cultures today. Consumers identify their coolness by seeking products and brands that provide them with cool status (Belk et al, 2010). While researchers have identified this cultural shift having a significant impact on how consumers are buying products, an in-depth understanding of its origin and meaning of this vernacular usage for the relevant consumer research is limited. As such, the purpose of this research is to understand consumers' 'cool seeking' behavior by exploring its possible determinants using a stepwise regression analysis. Drawing evidence from the Arab world our findings indicate that although the primary need for uniqueness drives cool identity, status concern is the most dominant predictor of cool-brand consciousness. We also demonstrate that in the Arab world cool is influenced by West, but not related to income, religion or gender.
\end{abstract}

The urge for uniqueness from the mainstream is the essence of coolness (CoolBrands, 2011). Literature suggests there are various manifestations of how individuals portray their 'coolness' behaviors. For example a person who exhibits novelty-oriented construction of himself, a rebellious trickster demeanor, emotionally self-protecting stance or one that has a 'cool' style of walking, gesturing and grooming can be termed as a cool person (Belk, 2006). Research indicates that such coolness is constantly sought by modern consumers to depict their character and lifestyle to others in their social network (Rahman and Cherrier, 2010). Traditionally, the element of cool is literally linked to teenagers as an ultimate approval of their peers (Danesi, 1994; O'Donnell and Wardlow, 2000) and to people in the fashion industry (Nancarrow et al., 2002); but contemporary consumers are not seen adhering to these age or gender stereotypes when seeking coolness (Neumeister,2006).

Despite previous studies in understanding 'coolness' (e.g. Belk, 2006) there is little quantitative research that examines 'cool seeking' phenomenon in its vernacular usage. This understanding is important because it can have specific effects on consumers purchase patterns. There is a general agreement that 'coolness' is conceptualized as alternative value system but an evidence based systematic linking between antecedents of coolness, demographic variables and cool seeking behavior has not been undertaken in the marketing literature. Thus, this study aims at providing a coolnessminded perspective of consumers to offer insights into the predictors of cool seeking tendency.

The present research provides a distinct psychological, social and behavioral patterns experienced by the Arab consumers when pursuing 'coolness' as part of their personality. The study highlights consumers' 'seeking coolness' is actually an extension of their 'cool identity' and their consequent preferences for cool products or 'cool brand consciousness'. 